\title{
The Evaluation Method for Augmented Reality Transformer under the Fusion Sets Pair Analysis and Generalized Evidence Theory
}

\author{
Shuangbai He ${ }^{1}$, Junfeng Li ${ }^{1}$, Xiao Liu ${ }^{1}$, Weixia Feng ${ }^{1}$, Manhua Wen ${ }^{1}$, Xiaoying Li ${ }^{2}$ \\ ${ }^{1}$ Education Training Evaluation Center, Guangdong Power Grid Co., Ltd, Guangzhou, 510520, China \\ ${ }^{2}$ Shaoguan Power Supply Bureau, Guangdong Power Grid Co., Ltd, Shaoguan, 512026, China
}

Keywords: Augmented reality transformer, Fusion Sets Pair, Generalized Evidence Theory

\begin{abstract}
Augmented reality technology is to integrate computer-generated three-dimensional virtual objects seamlessly with objects in real situations, thus providing a complementary and visual enhancement to the real world. In this paper, we set up a set of transformer evaluation index based on augmented reality. By comparing the calculation of the support and confidence of association rules, and at the same time the introduction of variable weight formula, get the weight coefficient of each evaluation index, effectively avoid the subjectivity of expert advice or experience; Based on the extensibility of the set pair analysis, the accuracy of the uncertainty factor of the transformer evaluation of the augmented reality is improved by using the 4-element relationship. But given the evaluation process is a series of uncertain factors, and generalized evidence theory fusion set pair analysis is presented of the augmented reality transformer evaluation methods, will be set after dealing with the true function of the results of analysis as the initial probability distribution of generalized evidence theory, the final step by step, to get the final result. Compared with the set analysis method, the augmented reality transformer evaluation method based on the generalized evidence theory has a good evaluation effect. But considering the uncertain factors during the evaluation process, and generalized evidence theory fusion set pair analysis is presented of the augmented reality transformer evaluation methods, will be set after dealing with the true function of the results of analysis as the initial probability distribution of generalized evidence theory, the final step by step, to get the final result. The comparative analysis shows, compared with the set analysis method, the augmented reality transformer evaluation method based on the generalized evidence theory has a good evaluation effect.
\end{abstract}

\section{The Establishment of the State Model Based on Augmented Reality Transformer Evaluation}

The Choice of State Quantity. The most fundamental thing to the augmented reality transformer evaluation is the establishment of evaluation system. On account of augmented reality transformer and the purpose \& features of the system application, through the research and analysis to the augmented reality transformer, we can establish the evaluation system from aspects of picture elements, picture texture and the robustness of the software. As shown in table 1: We choose adaptive lighting as augmented reality in the evaluation of transformer state because AR is on the basis of real device stack virtual model, and meantime the light of virtual model should be downy and it shouldn't conflict with real environment; we choose the actual state of the transformer as a reinforced reality is due to the fact that, for example, in the case of a real transformer superimposed model, it is necessary to look at the internal structure and to penetrate the visual display of the internal structure; select the precise overlay as augmented reality Transformer evaluates the state quantity because the current stack is only accurate on the xy plane, but the $\mathrm{Z}$ axis is not yet accurate. Satisfaction is defined as the relative relationship between the user's expectation and the actual use of the product after the user's demand is satisfied. The degree of intuition is defined as the sensibility of the user's direct contact 
with the product through specific maintenance activities. The degree of awareness; comfort is defined as the user from the physiological and psychological aspects of the satisfaction of the degree of satisfaction with the comprehensive evaluation, in the course of the use, mainly refers to wear augmented reality to wear the display system after the head and eyes Comfort degree

Division of Operating Status Levels. In the evaluation model of the existing reinforced real transformer, there is no uniform standard for the classification of the evaluation. In reference, this paper divides the evaluation grade of the transformer into four grades, namely, excellent, good, qualified.

In order to better reflect the evaluation of the transformer based on the enhancement of the reality and to reduce the interference of the subjective factors to the results, this paper chooses the five experts to evaluate the relay protection state respectively, the authoritative coefficients of $0.8,0.72,0.74,0.66,0.6$,

$$
S=\frac{\sum_{i=1}^{5} s_{i} w_{i}}{5}
$$

Where $s_{i}$ represents the score of each expert, and $w_{\mathrm{i}}$ represents the authority coefficient of each expert.

Determination of the Weight of the State Assessment Index. Where $x$ is the value of the evaluation index, $x_{\max }$ and $x_{\min }$ are the maximum and minimum values of the evaluation index $\mathrm{x}$. The curve of the variable weight function is shown in Figure 1.

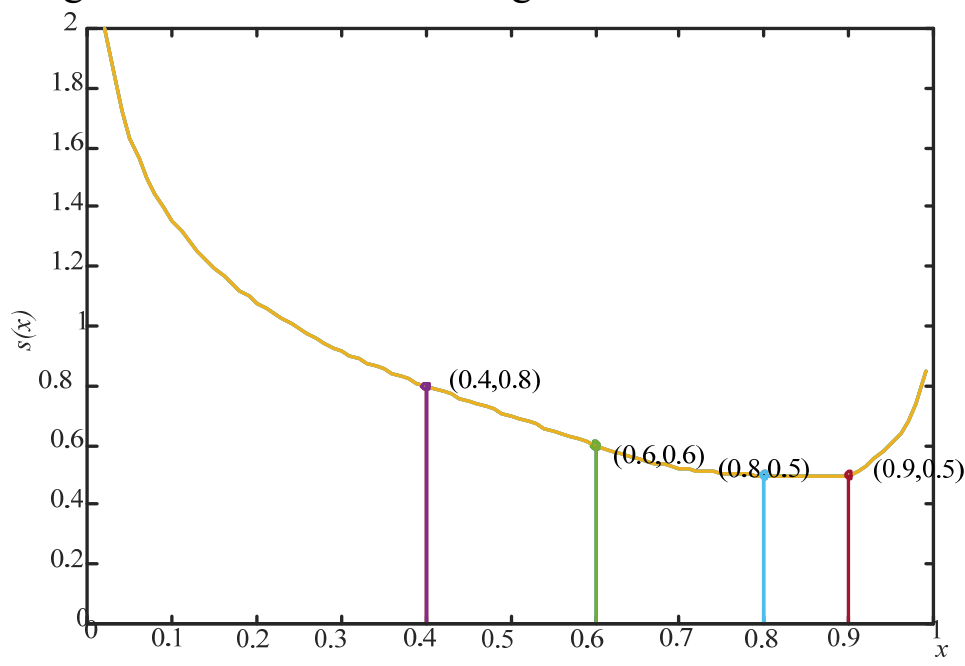

Figure 1. Curve of variable weight function

\section{Application of Enhanced Realistic Transformer Evaluation Based on Set Pair Analysis}

In the process of evaluating the actual transformer, the uncertainty of the result is determined, the uncertainty of the interval division, the establishment of the evaluation index evaluation and the uncertainty of the expert participation. Therefore, this paper adopts the cloud model instead of the fuzzy membership function, Fuzziness and randomness of the reliability assessment process.

But the definition of a normal cloud requires that a state has only one value, a "right and wrong", that is, expectation is a fixed value, but in real life, often more than one element belongs to this interval, that is expected to be a range, So the need to use trapezoidal cloud to describe [18], trapezoidal cloud pattern shown in Figure 2. 


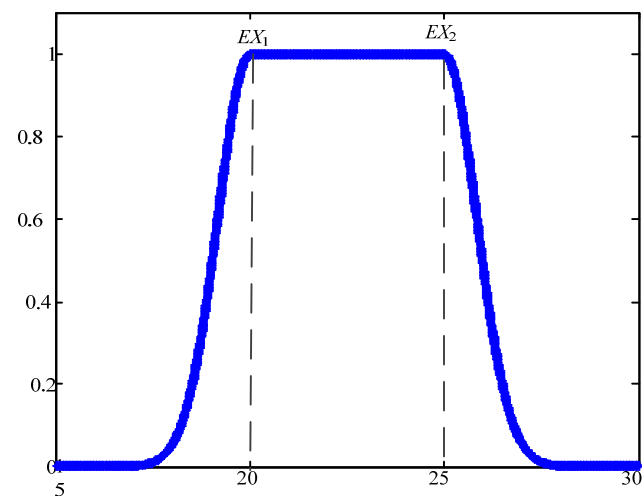

Figure 2. trapezoidal cloud pattern

The trapezoidal cloud model uses the expected values Ex1, Ex2, entropy En and super-entropy $\mathrm{He} 4$ digital features to convert between qualitative and quantitative data. To screen elements, for example, assume that the screen elements in four states, namely: "excellent", "good", "general", "unqualified." (ab) is in a certain state, the expression of the normal cloud of the picture element is

$$
r(x)=\left\{\begin{array}{rr}
\exp \left[-\left(x-E_{x 1}\right)^{2} /\left(2 E_{n}^{2}\right)\right] & x \leq E_{x 1} \\
1 & E_{x 1} \leq x \leq E_{x 2} \\
\exp \left[-\left(x-E_{x 2}\right)^{2} /\left(2 E_{n}{ }^{2}\right)\right] & x \geq E_{x 2}
\end{array}\right.
$$

And among them

$$
\begin{gathered}
\left\{\begin{array}{l}
E_{x 1}=a \\
E_{x 2}=b
\end{array}\right. \\
E_{n}=\frac{b-a}{6} .
\end{gathered}
$$

\section{Based on Generalized Evidence Theory State Assessment}

The Basic Concept of Generalized Evidence Theory. Let $U$ be the recognition framework of the open world, $U$ 's power set $2^{U}$ constitute the proposition set $2_{G}{ }^{U}, \forall A \in U$, if the function $m: 2^{U} \rightarrow[0,1]$ satisfies [20]

$$
\sum_{A \subseteq U} m_{G}(A)=1
$$

Then called $m$ is the generalized basic probability assignment (GBPA) on frame $U$. GBPA reflects the degree to which evidence supports the proposition $\mathrm{A}$ in the framework of identification, thus $m(A)$. The difference between GBPA and classical BPA is that GBPA has no limitations of $m(\Theta)=0$.

Generalized Evidence Theory Fusion Rule. In the generalized evidence theory, we set $\Theta 1$ $\cap \Theta 2=\Theta$, given two evidences, the synthetic rule is :

$$
\begin{gathered}
m(A)=\frac{(1-m(\Theta)) \sum_{B \cap C=A} m_{1}(B) m_{2}(C)}{1-k} \\
k=\sum_{B \cap C=\varnothing} m_{1}(B) m_{2}(C) \\
m(\Theta)=m_{1}(\Theta) m_{2}(\Theta)
\end{gathered}
$$

On $\mathbf{m}(\Theta)$ and Conflict - related Problems. The probability distribution of the traditional evidence theory uncertainty factor $m(\Theta)$ is zero, there is no physical meaning in the practical application, and the traditional evidence theory, because the importance of the distribution of evidence is not the same, it is easy to fall into evidence fusion

The situation of conflict. In the generalized evidence theory, the generalized basic probability assignment size of the empty set indicates the degree of support for the incomplete proposition of the 
identification framework. The larger the $\mathrm{m}(\Theta)$, the larger the representation of the system in the open world, the proposition in the identification framework is not as fast as possible ; If $m(\Theta)$ is small, it indicates that the system is in a closed world. The probability distribution of the uncertain factors $\Theta 1$ and $\Theta 2$ is the key to calculate the probability distribution of proposition $A$.

In order to ensure that the value of the uncertainty factor changes with the running state of the device, the confidence coefficient is defined by reference probability theory, and the confidence coefficient $\alpha_{r}(r=1,2 \ldots R)$ is introduced to modify the initial probability distribution and uncertainty The probability distribution of the factor. The weight of the above solution is substituted into the calculation of the confidence coefficient. On the one hand, the weight can be introduced into the evidence theory, so that the evaluation process is more realistic. On the other hand, the weight itself can reflect the size of the confidence and make the assignment objective.

$$
\begin{gathered}
m_{r}(H)=\alpha_{r} M_{r}(H) \\
m_{r}(\Theta)=1-\alpha_{r} \\
w_{r}^{\prime}=w_{r} / w_{\max } \\
\alpha_{r}=\alpha_{k} w_{r}^{\prime}
\end{gathered}
$$

Where $r$ is the total number of evidences, $M_{r}(H)$ is the result of fuzzy evaluation, $m_{r}(H)$ is the BPA of the modified BPA, $m_{r}(\Theta)$ is the uncertain factor, $\left\{w_{1}, w_{2}, \ldots w_{R}\right\}$ as evidence $\left\{f_{1}, f_{2}, \ldots f_{R}\right\}$, $\alpha_{k}$ is the confidence coefficient, take $0.9^{\prime}{ }_{\max }$ is the maximum value of $\left\{w_{1}, w_{2}, \ldots w_{R}\right\}$.

Decision Rules. Decision rules are critical to an inference system, and different decision rules can lead to different results. In this paper, the maximum basic probability distribution function rule is used to evaluate the target.

$$
\left\{\begin{array}{l}
m_{r}\left(H_{N_{0}}\right)-m_{r}\left(H_{N_{1}}\right)>\varepsilon_{0} \\
m_{r}(\Theta)<\varepsilon_{1} \\
m_{r}\left(H_{N_{0}}\right)>m_{r}(\Theta)
\end{array} .\right.
$$

\section{Calculating-examples analysis}

Evaluation of Reinforced Real Transformers Based on Set Pair Analysis. In the case of a real-life transformer in a region, the index of the relevant project is requested. The expert group will be asked to score the contents of the "cable laying" task, and the contents of the evaluation index system will be graded. Points to score. Assume that the weight of each expert is equal and the average of all expert ratings is used as the indicator implementation value. The results were shown in Table 1. 
Table 1 The expert rating of each evaluation indicator and the corresponding weight

\begin{tabular}{cccc}
\hline index & score & Constant weight & variable weight \\
\hline Picture element & 91.1 & 0.081 & 0.0836 \\
Picture texture ( texture, Material, color & 93.4 & 0.057 & 0.0603 \\
performance ) & & & 0.0783 \\
Intuitive & 88.6 & 0.078 & 0.1718 \\
Illumination consistency & 91.3 & 0.166 & 0.0662 \\
Geometric consistency & 84.6 & 0.069 & 0.0314 \\
Immersive experience & 81.6 & 0.034 & 0.0710 \\
Interactive expression & 87 & 0.072 & 0.0619 \\
Precise stacking & 91 & 0.06 & 0.0762 \\
Continuity of the system screen shows & 92.1 & 0.073 & 0.0281 \\
Occlusion of virtual reality & 91.9 & 0.027 & 0.0736 \\
Adaptive illumination & 91.5 & 0.071 & 0.0134 \\
The effectiveness of the design of the & 65.9 & 0.018 & 0.0844 \\
evaluation function & & & 0.0726 \\
Model refinement display & 84.6 & 0.088 & 0.0271 \\
Comfort & 81.1 & 0.079 & 0.027 \\
\hline
\end{tabular}

Calculate the constant weight of the evaluation index according to the cloud weighting calculation and the local penalty-incentive state variable weight function, as shown in Table 1.

Taking the picture element as an example, using the trapezoidal cloud, the membership of the picture element is $\mathrm{R}=\left[\begin{array}{llll}1 & 0 & 0 & 0\end{array}\right]$.

According to the formula (16),we can get results for $[0.3615,0.3399,0.2060,0.0926]$ based on the set of pairs of analytical methods enhance the reality of the transformer evaluation.

Enhanced reality Evaluation Based on Generalized Evidence Theory. The results of the analysis of each indicator set as the traditional evidence theory and the set pair analysis through the confidence function were used as the basic probability distribution result of the generalized evidence theory, the results were shown in Table 2 . In order to simplify the calculation, this paper selects the picture elements, picture texture and visualization as examples to illustrate the problem. $\mathrm{f} 1, \mathrm{f} 2$ and $\mathrm{f} 3$ represent the picture elements, picture texture and visuality respectively. $\mathrm{H} 1, \mathrm{H} 2, \mathrm{H} 3, \mathrm{H} 4$, represent excellent, good, general, unqualified respectively.

Table 2 Traditional evidence theory basic probability distribution calculation results

\begin{tabular}{ccccccc} 
Evidence & \multirow{2}{*}{$\mathrm{m}_{\mathrm{r}}(\Theta)$} & \multicolumn{9}{c}{$m_{\mathrm{r}}(\mathrm{H})$} & \multirow{2}{*}{ Judgment results } \\
\cline { 3 - 6 } & & $H_{1}$ & $H_{2}$ & $H_{3}$ & $H_{4}$ & \\
$f_{1}$ & 0 & 0.3672 & 0.3532 & 0.1923 & 0.0873 & $H_{1}$ \\
$f_{2}$ & 0 & 0.3155 & 0.3450 & 0.1967 & 0.1427 & $H_{2}$ \\
$f_{3}$ & 0 & 0.4169 & 0.31 & 0.2517 & 0.0214 & $H_{1}$ \\
\hline
\end{tabular}

Generalized evidence theory is more supportive of conclusions, the support for the normal state is $0.485,0.0827$ larger than the traditional 0.4023 , and the generalized evidence synthesis rate of $41 \%$, the traditional $21 \%$, Generalized evidence theory data fusion faster, this is the advantage of large data in the smart grid background, saving computing space and time;

In the fusion of $\mathrm{f} 1$ and $\mathrm{f} 2$ evidence of the time, the conflict coefficient of traditional evidence theory $\mathrm{k}=0.7814$, using the conflict coefficient of generalized evidence theory $\mathrm{k}=0.659$. This is because, according to the principle of maximum membership, the screen elements and intuition are in excellent condition; the picture texture is in a good state. At this time using traditional evidence theory to synthesize, the conflict is serious; this results in a synthetic error. However, using the generalized evidence theory, the confidence coefficient is used to assign the importance of each index to reduce the fusion of data.

Using the generalized evidence theory, if only fusion picture elements and picture texture, the probability reliability of the uncertain factor is assigned to 0.011 . If the three evaluation criteria are integrated, the probability reliability of the uncertain factor is assigned to 0.00168 . Therefore, the integration of multiple evidence enhances the reliability of the assessment and reduces the interference of uncertainties. 
Table 3 the set pair analysis and evidence theory comparison table

\begin{tabular}{ccccc}
\hline & Excellent & good & general & unqualified \\
\hline the set pair analysis & 0.3615 & 0.3399 & 0.2060 & 0.0925 \\
& & 0.3231 & 0.1489 & 0.059 \\
The traditional evidence theory & 0.4023 & & & \\
The generalized evidence theory & 0.4850 & 0.2192 & 0.1907 & 0.1051 \\
\hline
\end{tabular}

Table 3 shows, compared with the set pair analysis algorithm, the evidence theory has stronger support for the conclusion, for excellent, support for set pair analysis is 0.3615 , the support of the traditional evidence theory and generalized evidence theory is 0.4023 and 0.4850 . This is because, the set pair analysis method is to average the state assessment results, the support after the synthesis is always less than the maximum support before the synthesis; and due to the existence of polarization of synthetic rules, for mutual support evidence, Evidence theory synthesis results have a higher degree of credibility

\section{Conclusion}

1) Considering the serious distortion of the individual indicators and the relatively small weight in the evaluation of the reinforced concrete, it is proposed to use the variable weight theory to revise the evaluation index permanent, so that the weight of the distortion module can be increased. Which facilitates system developers and users to quickly find distortion modules and improve the corresponding modules.

2) For the uncertainty in the process of assessing the credibility of the uncertainty, using the trapezoidal cloud model to solve the membership degree, combine the randomness and ambiguity of the evaluation process, expand the description interval of uncertainty and improve the accuracy of the assessment results.

3) Using the general theory of evidence, taking full account of a series of uncertainties in the evaluation, layer-by-layer fusion, to obtain a realistic evaluation of the transformer. Finally, an example shows that the fusion results of the generalized evidence is more reliable than the traditional evidence theory and set pair analysis.

\section{Acknowledgments}

This study was sponsored by the Virtual Reality and Augmented Reality Key Laboratory of Guangdong Power Grid Co., Ltd

\section{References}

[1] Barry Crabtree, Bradley Rhodes. Wearable computing and the remembrance agent [J]. BT Technology Journal. 1998, 16(3):118-124.

[2] Bowman D, Gabbard J1, Hix DA. A survey of usability in virtual environments: classification and comparison of methods [J]. Presence: Teleoperators and Virtual Environments, 2008, 11(4): 404-424.

[3] Zeng Weiping, Yu Chun, Wu Zhipeng, et al. Design and development of virtual maintenance human model state comfort assessment library [J]. Helicopter technology, 2014(4):39-44.

[4] Zhao Caishan, Sun Guanghui, Liang Dongming, Research on simulation credibility evaluation of rural power distribution simulation training system $[\mathrm{J}]$. China rural water conservancy and hydropower, 2014(12):169-172. 